\title{
ARTICLE OPEN Amplitudon and phason modes of electrocaloric energy interconversion
}

\author{
John Mangeri ${ }^{1}$, Krishna C Pitike ${ }^{2}$, S Pamir Alpay ${ }^{1,2}$ and Serge Nakhmanson ${ }^{1,2}$
}

Solid-state electrothermal energy interconversion utilising the electrocaloric effect is currently being considered as a viable source of applications alternative to contemporary cooling and heating technologies. Electrocaloric performance of a dielectric system is critically dependent on the number of uncorrelated polar states, or 'entropy channels' present within the system phase space. Exact physical origins of these states are currently unclear and practical methodologies for controlling their number and creating additional ones are not firmly established. Here we employ a multiscale computational approach to investigate the electrocaloric response of an artificial layered-oxide material that exhibits Goldstone-like polar excitations. We demonstrate that in the low-electric-field poling regime, the number of independent polar states in this system is proportional to the number of grown layers, and that the resulting electrocaloric properties are tuneable in the whole range of temperatures below $T_{C}$ by application of electric fields and elastic strain.

npj Computational Materials (2016) 2, 16020; doi:10.1038/npjcompumats.2016.20; published online 15 July 2016

\section{INTRODUCTION}

Electrocaloric (EC) effect is defined as the variation of the dielectric material entropy as a function of the electric field at a given temperature, which results in an adiabatic temperature change. Recently, there has been significant progress ${ }^{1-3}$ in the development of polar (i.e., possessing spontaneous polarisation) dielectrics that display large EC temperature shifts $\Delta T$ under electric-field poling. These systems include a variety of ferroelectric ceramics, ${ }^{4-6}$ polymers ${ }^{7-10}$ and liquid crystals. ${ }^{11}$ A few relaxor and antiferroelectric materials may exhibit negative EC effect, which is especially advantageous for solid-state refrigeration. $^{12}$ The best values of positive EC $\Delta T$ in modern nanoengineered materials range from 20 to $45 \mathrm{~K}$, for electric-field sweeps $\Delta E \geqslant 500 \mathrm{kV} / \mathrm{cm}^{1-6,13,14}$ whereas negative $\mathrm{EC} \Delta T$ remain below $-10 \mathrm{~K}$ for much smaller $\Delta E^{15-18}$

The magnitude of the EC $\Delta T$ is proportional to a logarithm of the number of possible polar states, or independent 'entropy channels' in the system. ${ }^{3,19}$ Therefore, it is highly desirable to acquire precise understanding of physical phenomena underpinning the emergence of these states and develop practical methods for engineering additional entropy channels into dielectrics. Moreover, since entropic changes involving evolution of other ferroic order parameters can be cumulative with the EC effect, more advanced multicaloric materials concepts blending polar, magnetic and elastic energy-interconversion functionalities, are also being considered. ${ }^{20-24}$

Here we use a multiscale computational approach that combines $a b$ initio quantum mechanical simulations, phenomenological Landau theory and thermodynamical evaluations to investigate the EC response of a 'material template' based on a quasi-two-dimensional system that exhibits polar Goldstone-like ${ }^{25-27}$ excitations. This template system is an ' $n=2^{\prime}$ Ruddlesden-Popper (RP) type ${ }^{28} \mathrm{PbSr}_{2} \mathrm{Ti}_{2} \mathrm{O}_{7}$ (PSTO) layered-oxide superlattice shown in Figure 1a. Such or similar materials have been successfully grown, e.g., by utilising molecular-beam epitaxy synthetic techniques. ${ }^{29-31}$

The most interesting predicted feature of this system is that under biaxial basal-plane strains ranging from slight tensions (up to $0.2 \%$ ) to modest compressions (up to $2 \%$ ) it loses crystalline anisotropy with respect to the direction of its spontaneous polarisation $\mathbf{P}=\left(P_{x}, P_{y}\right)_{1}{ }^{32}$ whereas rocksalt-type $\mathrm{SrO}-\mathrm{SrO}$ inserts in between perovskite slabs prevent any polarisation in the out-ofplane direction $z$. The system free-energy density landscape $f\left(P_{x}, P_{y}\right)$ is shown in Figure $1 \mathrm{~b}$ and has a distinctive sombrero-hat shape characteristic of a presence of a Goldstone-like or phason excitation. ${ }^{25-27}$ In the case of PSTO, that excitation manifests itself as an easy-i.e., requiring almost no consumption of energy-rotation of $\mathbf{P}$ along the minimum-energy path within the basal plane, whereas its amplitude is kept constant.

This path, shown in greater detail in Figure 1c for zero biaxial strain, is only approximately circular-hence the '-like' suffix above, as for the true Goldstone excitation it has to be a perfect circle. Here the absolute energy minima (at $-6.26 \mathrm{meV}$ per structural unit, or s.u.) are located along the [100], $\theta=0^{\circ}$, and three symmetrically equivalent crystallographic directions, and separated by saddle points (at $-6.01 \mathrm{meV} / \mathrm{s}$.u.) located along [110], $\theta=45^{\circ}$, and symmetrically equivalent directions. The competition between the [100]- and [110]-oriented sets of energy extrema can be controlled by applied strain; e.g., a small amount of tension can shift the minima to the [110] set of directions and saddle points to the [100] set.

\section{RESULTS}

We have developed Landau-type polynomial expansions for $f$ from $a b$ initio calculations, ${ }^{32}$ including couplings to elastic strain and 
a

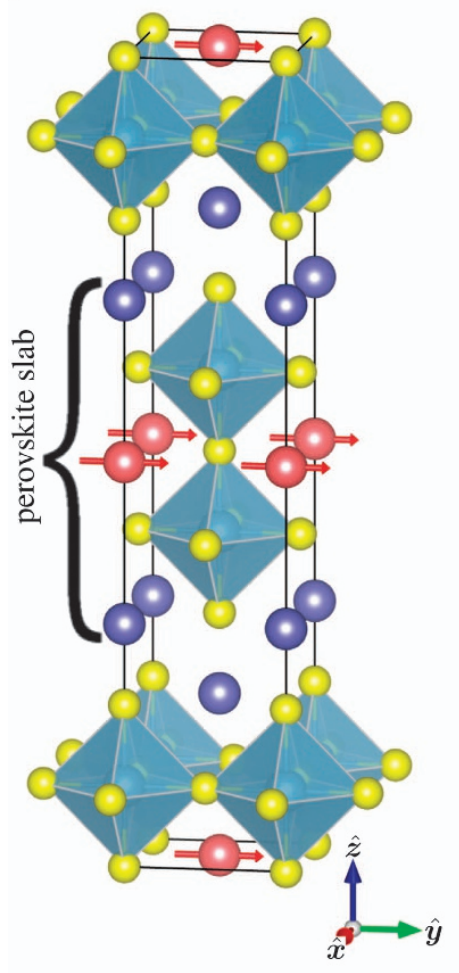

b

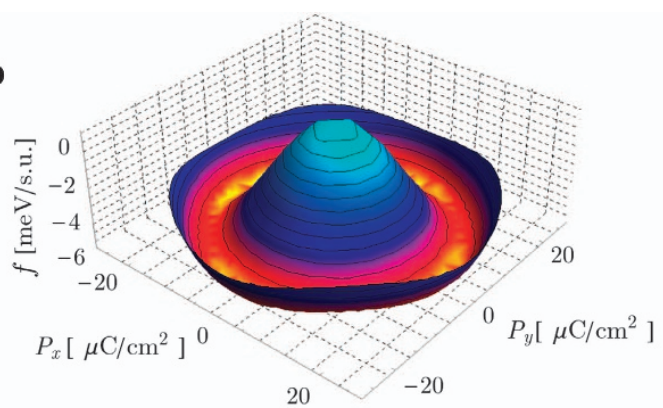

C

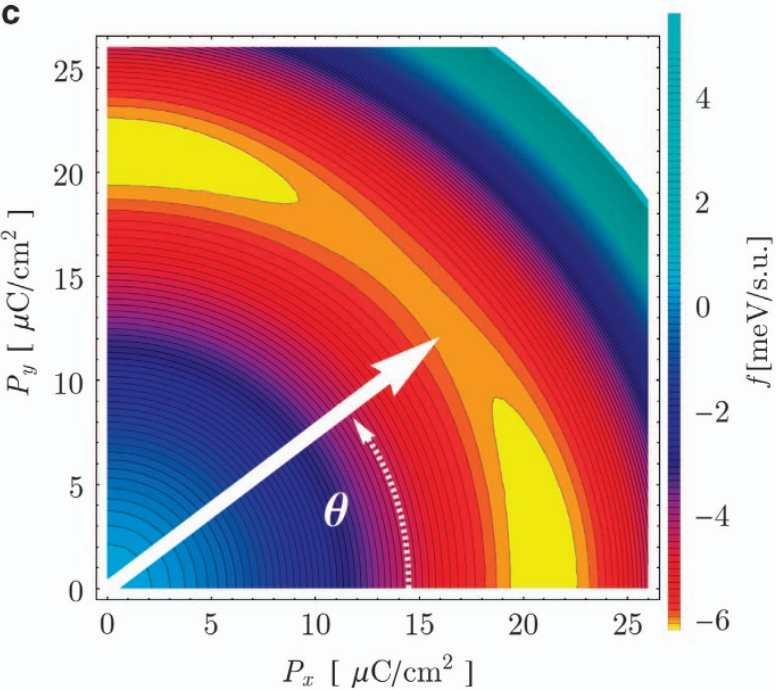

Figure 1. Crystal structure of $\mathrm{PbSr}_{2} \mathrm{Ti}_{2} \mathrm{O}_{7}$ and Goldstone-like energy surface. (a) Tetragonal unit cell consisting of two slabs connected by a body-centring translation (space group $14 / \mathrm{mmm}$ ). $\mathrm{Pb}$ and $\mathrm{Sr}$ atoms are shown in red and blue, respectively, while the $\mathrm{TiO}_{6}$ cages are represented by light-blue semitranslucent octahedra. (b) Three-dimensional and (c) contour-map views of the system energy density function $f$ with respect to developing in-plane polarisation $\mathbf{P}=\left(P_{x}, P_{y}\right)$ at $\varepsilon \sim 0 \%$. The energy of the paraelectric phase is taken as zero. The location of the polarisation vector within the minimum-energy 'groove' and its azimuthal angle $\theta$ are marked out in c for the shown $P_{x}, P_{y}>0$ quadrant of the energy surface.

applied electric field E. Since RP structure does not support out-of-plane polar distortions, ${ }^{32,33}$ only the in-plane polarisation components were included. Also, only planar biaxial strain-tensor components $\varepsilon_{x x}=\varepsilon_{y y} \equiv \varepsilon$, accounting for the epitaxial misfit strain on a square substrate, were considered in the expansion. Both linear and quadratic couplings involving $\varepsilon$ were included to reproduce polarisation locking-unlocking phase transition under changing strain. With all the simplifications, this quasi-twodimensional free-energy function is

$$
\begin{aligned}
f= & a_{1}\left(T-T_{C}\right)\left(P_{x}^{2}+P_{y}^{2}\right)+a_{2}\left(P_{x}^{4}+P_{y}^{4}\right)+a_{3} P_{x}^{2} P_{y}^{2} \\
& +a_{4}\left(P_{x}^{6}+P_{y}^{6}\right)+a_{5}\left(P_{x}^{4} P_{y}^{2}+P_{x}^{2} P_{y}^{4}\right) \\
& +\left(X_{1}\left(P_{x}^{2}+P_{y}^{2}\right)+x_{2}\left(P_{x}^{4}+P_{y}^{4}\right)+X_{3} P_{x}^{2} P_{y}^{2}\right) \varepsilon \\
& +\left(X_{4}\left(P_{x}^{2}+P_{y}^{2}\right)+X_{5}\left(P_{x}^{4}+P_{y}^{4}\right)+X_{6} P_{x}^{2} P_{y}^{2}\right) \varepsilon^{2}-P_{x} E_{x}-P_{y} E_{y} .
\end{aligned}
$$

The estimated system $T_{C} \simeq 120 \mathrm{~K}$, while the rest of the expression coefficients are given in Supplementary Information.

The equilibrium state of the system at a given temperature $T$ and strain $\varepsilon$ is determined by minimising $f$ with respect to the polarisation vector components $P_{x,} P_{y}$. System excess heat capacity $^{34} \Delta C$ and pyroelectric coefficients $p_{\gamma}, \gamma=x, y$, can be obtained from the equilibrium values of polarisation $\left(\mathbf{P}^{0}\right)$ and energy density $f^{0} \equiv f\left(\mathbf{P}^{0}\right)$ as

$$
\begin{aligned}
& \Delta C=-T\left(\partial^{2} f^{0} / \partial T^{2}\right)_{\mathbf{E}, \varepsilon}, \\
& p_{\gamma}=\left(\partial S / \partial E_{\gamma}\right)_{\varepsilon, T}=\left(\partial P_{\gamma}^{0} / \partial T\right)_{\varepsilon, \mathbf{E}} .
\end{aligned}
$$

Then the adiabatic change in the system temperature $\Delta T$ under the influence of varying applied electric field $\mathbf{E}$ is ${ }^{35}$

$$
\Delta T=-T \sum_{\gamma=x, y} \int_{E_{\gamma, a}}^{E_{\gamma, b}} d E_{\gamma}\left[\frac{p_{\gamma}}{C+\Delta C}\right]_{\varepsilon, \mathbf{E}, T},
$$

where $E_{\gamma, b}-E_{\gamma, a} \equiv \Delta E_{\gamma}, E_{\gamma, a} \leqslant E_{\gamma, b}$, are changes in the $x$ and $y$ components of $\mathbf{E}$ during the poling procedure, and $C(T)$ is the system heat capacity. Equations (1)-(4) were then used to study polarisation switching and the resulting temperature change $\Delta T$ in PSTO under applied field E. More details on the evaluation of both heat capacity terms and their influence on the magnitude of $\Delta T$ are provided in the Materials and Methods section below and in Supplementary Information.

Remarkably different types of the system polarisation behaviour are observed under conditions of either high-, or low-field poling. In the former case, i.e., for $\Delta E_{\gamma} \geqslant 40-50 \mathrm{kV} / \mathrm{cm}$ (depending on the value of $\varepsilon$ ), energy landscape shown in Figure 1 is deformed away from its original sombrero-hat shape. Application of a large 
a

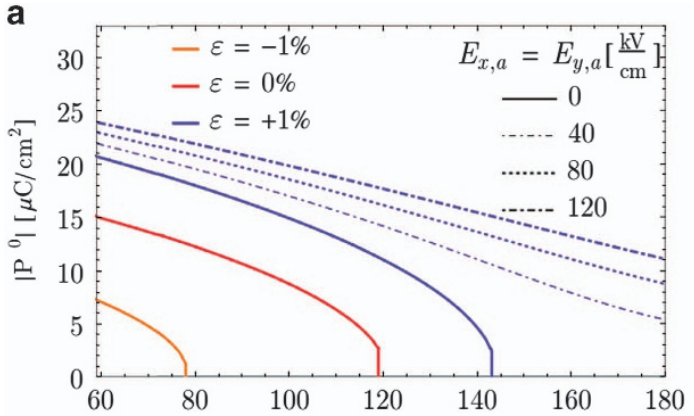

b

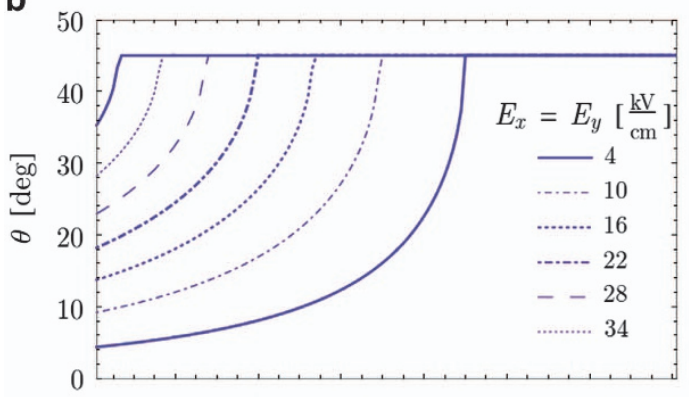

C

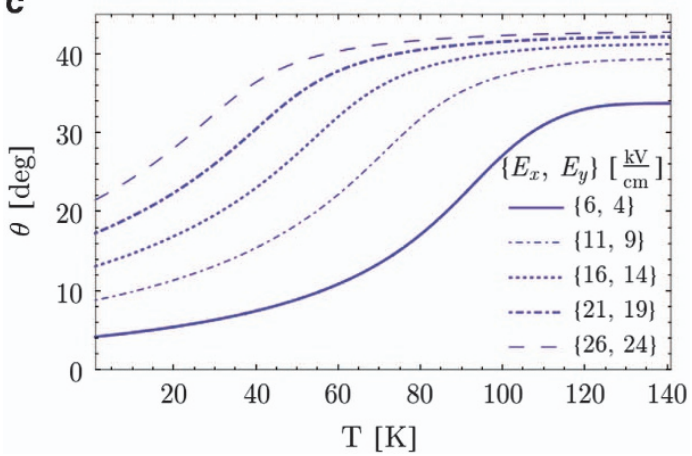

Figure 2. Amplitudon versus phason polarisation switching. (a) Temperature dependence of the zero-field polarisation amplitude at different biaxial strains. For $\varepsilon=1 \%$, the same dependence is shown in dashed lines for applied fields ranging from 40 to $120 \mathrm{kV} / \mathrm{cm}$. Temperature dependence of the polarisation azimuthal angle (or phase) $\theta$ under (b) symmetrically, $E_{x, a} \equiv E_{y, a}$ and (c) asymmetrically, $E_{x, a} \neq E_{y, a}$ applied static electric fields. In the former case, the polarisation vector abruptly locks with the direction of the field at some specific temperature $T_{\text {lock}}$, while in the latter case it smoothly aligns with the field.

electric field $\left(E_{x, a}, E_{y, b}\right)$ creates a deep energy minimum along its direction, locking the polar vector $\mathbf{P}^{0}$ inside this minimum and destroying the Goldstone-like excitation. Poling field $\left(\Delta E_{x}, \Delta E_{y}\right)$ then preserves the locked state of the polarisation. If the direction of $\mathbf{E}$ remains fixed during the poling, polarisation azimuthal angle $\theta$ stays constant, while only the amplitude $\left|\mathbf{P}^{0}\right|$ changes value. Such amplitudon mode of polarisation switching is usually seen in conventional EC materials, ${ }^{5,6,34,35}$ where the largest variations in $\left|\mathbf{P}^{0}\right|$ and thus the largest changes in $p_{\gamma}$ and $\Delta T$ occur near $T_{C}$.

In Figure $2 a$, we present the $\left|\mathbf{P}^{0}\right|^{2}$ versus $T$ dependence at different values of $\varepsilon$ (see Supplementary Figure 1 for more details). This includes $\varepsilon=1 \%$, where even at zero field the Goldstone-like excitation disappears in favor of a localised minimum along [110]. These results display transitional behaviour typical for a generic perovskite ferroelectric around $T_{C} \cdot{ }^{36}$ Curves for a number of different applied fields $E_{x, a}=E_{y, a}$ are also shown for $\varepsilon=1 \%$ and, as expected, show a persistence of the polar phase beyond the zero-field $T_{C}$. For the poling field $\Delta E_{\gamma} \sim 200 \mathrm{kV} / \mathrm{cm}$, we obtain the values of $\Delta T$ in the range of $1-2 \mathrm{~K}$ (Supplementary Figure 2), which is similar to the performance of conventional EC materials undergoing amplitudon-polarisation switching. ${ }^{5,6,34,35}$

In the case of low-field poling, i.e., for $E_{\gamma, a} \sim 1-5 \mathrm{kV} / \mathrm{cm}$ and $\Delta E_{\gamma} \leq 10-30 \mathrm{kV} / \mathrm{cm}$, the sombrero-hat energy landscape is only slightly perturbed. If the $\mathbf{P} \|[100]$ state of Figures $1 \mathrm{~b}$ and $\mathrm{c}$ is taken as a starting point, applying noncollinear $\mathbf{E}$ induces a rotation of the polar vector along the minimum-energy groove until $\mathbf{P}|| \mathbf{E}$. During this rotation process, which we refer to as phason polarisation switching, its azimuthal angle $\theta$ changes while its amplitude $\left|\mathbf{P}^{0}\right|$ remains approximately constant.

The $\theta(T)$ dependence for a number of different magnitudes of $\mathbf{E}$ is shown in Figure $2 \mathrm{~b}$ and Figure $2 \mathrm{c}$ for symmetric, $E_{x, a} \equiv E_{y, a}$ and asymmetric, $E_{x, a} \neq E_{y, a}$, static applied fields, respectively. Here, the polarisation is initially pointing towards the nearest energy minimum, so that, e.g., $\theta=0^{\circ}$, while the $\widehat{\mathbf{P}, \mathbf{E}}$ angle is $<45^{\circ}$. In the both cases, it is observed that $\mathbf{P}$ can align with $\mathbf{E}$ only if sufficient energy is provided to the system in the form of heat. The temperature value at which the alignment happens $\left(T_{\text {lock }}\right)$ can be adjusted by changing the magnitude of $\mathbf{E}$ throughout the whole temperature interval $\left(0 \rightarrow T_{C}\right)$, similarly to how the value of $T_{C}$ can be attuned by the applied strain $\varepsilon$ during the amplitudon switching. However, for asymmetrically applied fields, the alignment of $\mathbf{P}$ with $\mathbf{E}$ always happens smoothly (see Figure $2 \mathrm{c}$ ), not resulting in an emergence of large $p_{y}$ in the vicinity of $T_{\text {lock. }}$. On the other hand, for symmetrically applied fields, polarisation alignment occurs abruptly (see Figure 2b; Supplementary Figure 3 ) and produces large pyroelectric response, which is strikingly similar to the behaviour of $\left|\mathbf{P}^{0}\right|$ near $T_{C}$ during the high-field poling. The application of sufficiently large $\mathbf{E}-$ which is dependent on $T$-along [110] lowers the energy of the associated saddle point until it becomes the new energy minimum, while simultaneously raising the energy of the original minima along [100] and [010]. This creates a strong bias for locking $\mathbf{P}$ along the saddle-point direction, while such an incentive would be missing in the case of asymmetrically applied fields.

Since passage of the applied field through the saddle-point direction is accompanied by an emergence of large pyroelectric coefficients, which should in turn result in large EC $\Delta T$, the following simple poling scenario can be considered. Starting with the polarisation along the $x$ direction, $\theta=0^{\circ}$, a static field $E_{x} \equiv \tilde{E}$ is applied, i.e., $\Delta E_{x}=0$ which eliminates one of the two integrals in Equation (4). Then, the field along the $y$ direction is changed from $E_{y, a}<\tilde{E}$ to $E_{y, b}>\tilde{E}$. This situation is illustrated step by step in Figure 3, which suggests that even for modest poling-field ramps $\Delta E_{y}$, bracketing the value of $\tilde{E}$, it is possible to trigger rotation of $\mathbf{P}$ by an angle close to $90^{\circ}$, e.g., from the [100] energy minimum, through the saddle point along [110] and into the [010] energy minimum. The $\theta(T)$ plot in Figure $3 \mathrm{~b}$ shows that $E_{y, b}<\tilde{E}$ initiates a smooth alignment of $\mathbf{P}$ with $\mathbf{E}$, while only $E_{y, b}>\tilde{E}$ results in an abrupt switching of $\mathbf{P}$ between the two neighbouring basins within the energy landscape, after which it again smoothly aligns with $\mathbf{E}$ as $T$ is raised above $T_{\text {lock}}$.

Figure $3 c$ shows that abrupt changes in $\theta$ translate into large negative values of EC $\Delta T$. As illustrated by the two different switching cases, the position of the phason EC peak on the temperature axis, as well as its width, can both be controlled purely by means of applied electric field-specifically by setting the values of $\tilde{E}$ and $\Delta E_{y}$, respectively. For example, changing $\tilde{E}$ from 5 to $13 \mathrm{kV} / \mathrm{cm}$ moves the center of the phason EC peak down from 60 to $30 \mathrm{~K}$. Such precise tuning of the shape and location of the phason-induced EC response can be accomplished for all temperatures below $T_{C}$. Typical maximum entropy changes achieved in this poling scenario are $0.5-1 \mathrm{~J} / \mathrm{kg} / \mathrm{K}$ (Supplementary Figure 4), similar to values observed by others for low-field switching. ${ }^{37}$

The negative sign of the phason-switching induced $\Delta T$ originates from the following consideration: as $T \rightarrow T_{\text {lock }}$ and 

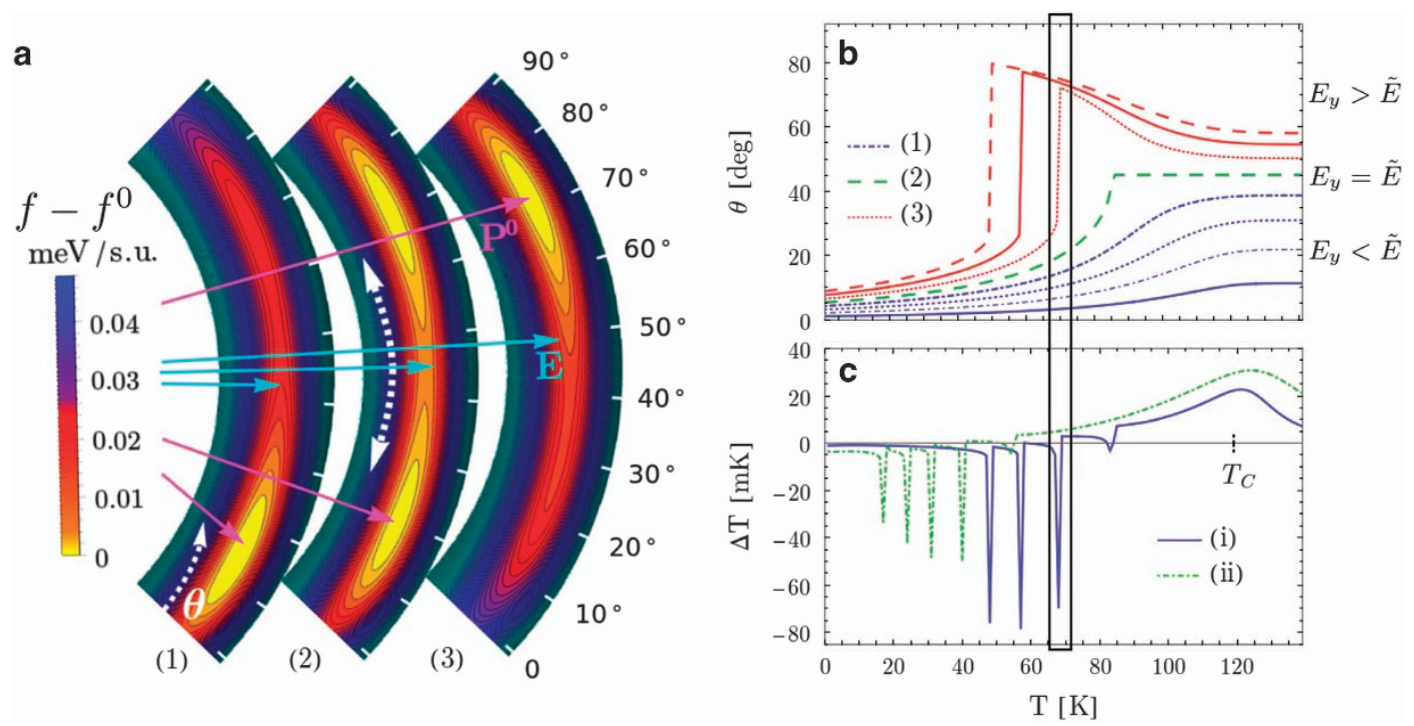

Figure 3. Phason-induced polarisation rotation and the resulting EC temperature change. (a) A step-by-step sketch of polarisation rotation under poling electric field $E_{y}$ at $\tilde{E}=5 \mathrm{kV} / \mathrm{cm}$ and $T=68 \mathrm{~K}$. Energy-surface slices marked (1) through (3) correspond to the following states of the system: (1) $\mathbf{P}$ in vicinity of the minimum along [100], $\theta \sim 0^{\circ}, E_{y}<\tilde{E} ;(2) \mathbf{P}$ passing through the saddle point along [110], $\theta \sim 45^{\circ}$, for $E_{y} \simeq \tilde{E}$; (3) $\mathbf{P}$ switched into the equivalent minimum along [010], $\theta \sim 90^{\circ}, E_{y}>\tilde{E}$. Arrows represent approximate directions of $\mathbf{P}^{0}$ and $\mathbf{E}$ at steps (1) through (3). (b) $\theta(T)$ dependence under applied static electric field $\left(\tilde{E}, E_{y}\right)$ where (1) $E_{y}<\tilde{E}$, (2) $E_{y} \simeq \tilde{E}$ and (3) $E_{y}>\tilde{E}$. Only in the latter case polarisation can switch from one energy minimum to the next, giving rise to a peak in the EC response. The complete poling sequence consists of sweeping $E_{y}$ from (1) to (3) through (2) at fixed $T$. (c) Examples of the phason-induced EC response generated by this procedure highlight its tuneability: (i) $\tilde{E}=5 \mathrm{kV} / \mathrm{cm}$ and $\Delta E_{y}=7 \mathrm{kV} / \mathrm{cm}$, (ii) $\tilde{E}=13 \mathrm{kV} / \mathrm{cm}$ and $\Delta E_{y}=17 \mathrm{kV} / \mathrm{cm}$. The connection between an abrupt change of $\theta$ and the produced EC response (at some fixed $E_{y}>\tilde{E}$ ) is outlined by the rectangular selection spanning panels (b, $\mathbf{c}$ ).

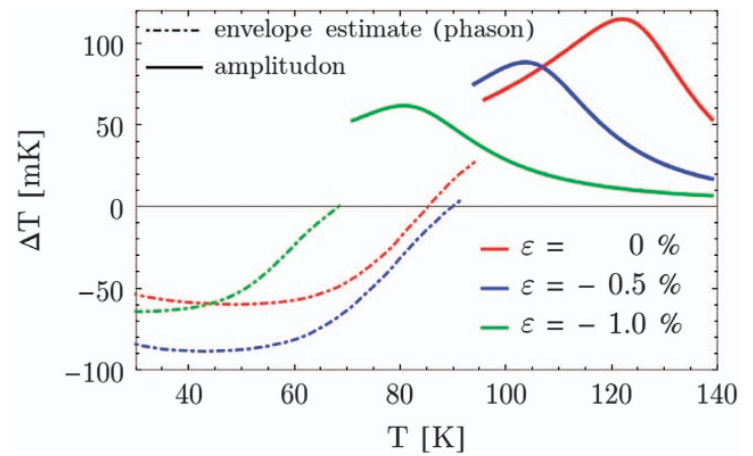

Figure 4. Phason and amplitudon polarisation-switching contributions to the EC temperature change at different strains. All the presented curves are computed for $\tilde{E}=5 \mathrm{kV} / \mathrm{cm}$ and $\Delta E_{y}=34 \mathrm{kV} / \mathrm{cm}$. Note that for $T$ near $70 \mathrm{~K}$ it should be possible to flip the sign of the EC response (i.e., switch from cooling to heating, or vice versa) by tuning the value of $T_{C}$ by applied strain.

polarisation rotation occurs, the $\widehat{\mathbf{P}, \mathbf{E}}$ angle is always diminished. Naturally, polarisation component $P_{\gamma, b}$ after the rotation is always larger than component $P_{\gamma, a}$ before the rotation, where $\gamma$ is the direction along the field. Thus, the associated $p_{\gamma} \sim\left(P_{\gamma, b}-P_{\gamma, a}\right) /\left(T_{\text {lock }}-T\right)$ is positive, which results in negative $\Delta T$ according to Equation (4).

As shown in Figure $3 c$, abrupt changes of $\theta$, each occurring under a specific fixed value of $E_{y}>\tilde{E}$, result in an emergence of sharp bumps in the EC $\Delta T$ response. Furthermore, by choosing the values of the stationary $\tilde{E}$ field and the bracketing $\Delta E_{y}$ poling interval, these peaks can be shifted to lower or higher temperatures on demand. When during the poling procedure $E_{y}$ is swept continuously from $E_{y, a}<\tilde{E}$ to $E_{y, b}>\tilde{E}$, individual peaks in the $\Delta T$ curve merge into an envelope that is presented in Figure 4 for different values of $\varepsilon$ (see also Supplementary Figure 5).
Remarkably, the variation of $\varepsilon$ by $\leqslant 1 \%$ can result in the system EC response changing from cooling (phason contribution) to heating (amplitudon contribution) at the same operating temperature.

\section{DISCUSSION}

The EC temperature changes in PSTO in the low-field poling regime range from -50 to $-100 \mathrm{mK}$, i.e., they are two orders of magnitude smaller compared to the (positive) $\Delta T$ values produced during the conventional, high-field poling. However, since, without the loss of generality, the quasi-two-dimensional form of $f$ can describe the behaviour of a single slab whose polarisation is decoupled from those of its neighbours, cumulative EC $\Delta T$ in a multi-slab system should look very different for the high- and low-field poling cases. It is noteworthy that the approximation of electrically decoupled polar slabs is already quite good for bulk PSTO, as illustrated by an absence of phonon-band dispersion for polar modes in the direction perpendicular to the slab planes; see Figure 2 in reference 32 .

In the high-field regime, individual slab polarisations are switched 'all at once,' being forcefully correlated by the applied field. That is, the multi-slab system possesses only one entropy channel and the value of $\Delta T \simeq 1-2 \mathrm{~K}$ quoted above for PSTO should represent its aggregate EC response. In the low-field regime, applied fields are insufficient to correlate the directions of (disordered) polar vectors in different slabs and, therefore, polarisation rotations under the cycling of the field should occur independently in each slab. In such a case, each slab acts as a separate entropy channel and the aggregate EC response of the whole system should be proportional to the logarithm of the number of slabs. Then, even with individual slab contributions being low ( 100 mK for PSTO), a system comprised of a large number of slabs should possess an aggregate $\Delta T$ that is at least comparable with other state-of-the-art negative EC materials. ${ }^{15-18}$

The estimate of one entropy channel per slab is conservative, as in the model presented here any influence of polar domain-wall 
dynamics on the EC response is not taken into account, i.e., each slab is considered to be in a monodomain state. An investigation of polarisation-closure patterns in PSTO nanostructures predicted that these patterns are likely to form and their behaviour sensitively depends on the applied strain, $^{38}$ suggesting that multiple entropy channels may be created (or destroyed, if needed) in each slab by distorting its shape. Alternatively, a nanoisland device geometry, where polar states of individual islands uncorrelated from each other, can be used instead of a continuous-film one.

In summary, we have shown that layered polar systems with Goldstone-like instabilities should exhibit attractive EC properties that are highly tunable by applying electric fields and elastic distortions over a wide range of operating temperatures below $T_{C}$, which may even include on-demand switching between cooling and heating. By virtue of operating at low electric fields, device applications of actual materials will require modest power consumption, compared with most other known EC compounds, where fields of upwards of $500 \mathrm{kV} / \mathrm{cm}$ are needed for best performance. Furthermore, in such model systems the mechanisms behind the emergence of independent entropy channels can be clearly established allowing for an easy up- or down-scaling of the system entropy-flow and EC characteristics by growing an appropriate number of layers. Although for the specific example of PSTO, a single monodomain-slab EC response in the low-field operation is only around $0.1 \mathrm{~K}$, it should be possible to employ 'materials by design' principles to develop new compounds with improved EC properties. The competition between the [100]- and [110]-oriented sets of energy minima in polar-perovskite layers, leading to Goldstone-like excitations, appears to be a generic geometrical feature of such systems, rather than a peculiar trait distinctive to PSTO. We have recently identified other layeredoxide structures that should possess similar properties. ${ }^{39}$

\section{MATERIALS AND METHODS}

$A b$ initio calculations were performed with the density-functional theory code Quantum Espresso ${ }^{40}$ utilising local-density approximation in the Perdew-Zunger parametrisation ${ }^{41}$ and Vanderbilt ultrasoft pseudopotentials. ${ }^{42}$ Vibrational frequencies, ionic displacement patterns and system vibrational density of states, were obtained using densityfunctional perturbation theory approach within Quantum Espresso. ${ }^{43}$ The system total heat capacity $C(T)$, used in Equation (4) above, was evaluated from the vibrational density of states ${ }^{44,45}$ computed for the non-polar system configuration (see Supplementary Information and Supplementary Figure 6 for more details).

Ionic forces were relaxed to less than $0.2 \times 10^{-5} \mathrm{Ry} / \mathrm{bohr}$ $\left(\sim 0.5 \times 10^{-4} \mathrm{eV} / \AA \AA \AA\right)$ and the appropriate stress tensor components $\sigma_{\alpha \beta}$ ( $a$ and $\beta$ are Cartesian directions $x, y$ and $z$ ) were converged to values below 0.2 kbar. Epitaxial thin-film constraint on a cubic (001)-oriented substrate was simulated by varying the in-plane lattice constant $a$ of the tetragonal cell and allowing the out-of-plane lattice constant $c$ to relax (by converging the normal stress in this direction to a small value), while preserving the imposed tetragonal unit-cell symmetry. The biaxial misfit strain $\varepsilon$ is defined as $\left(a-a_{0}\right) / a_{0}$, where $a_{0}$ is the in-plane lattice parameter of the free standing PSTO structure with all the normal stress tensor components relaxed to $<0.2 \mathrm{kbar}$.

lonic Born effective-charge tensors $Z_{i, \alpha \beta}^{*}$, where $i$ is the ion number, and high-frequency dielectric permittivity tensor $\varepsilon_{\alpha \beta}^{\infty}$ were calculated by utilising the density-functional perturbation theory approach. The system polarisation was evaluated with a linearised approximation ${ }^{46}$ involving products of $Z_{i, \alpha \beta}^{*}$ and ionic displacements away from centrosymmetric positions. The value of $T_{C}(\sim 120 \mathrm{~K})$ was estimated from energy differences between the non-polar $14 / \mathrm{mmm}$ and polarised $\mathbf{P} \|$ [100], and $\mathbf{P} \|[110]$ states of the system.

We should point out that due to the purely analytical nature of our approach it does not account for spatial inhomogeneities of the polarisation $\mathbf{P}$ and elastic-field $\varepsilon_{\alpha \beta}$ variables. Such inhomogeneities can be present in epitaxially clamped thin films producing additional contributions to the aggregate $\Delta T$ that stem from, e.g., elastocaloric effects and polydomain behaviour. Although we have not yet studied piezoelectric response of PSTO in detail, we expect most of its piezoelectric coefficients to be low-with the same being true about the magnitude of the intrinsic elastocaloric effect-as polarisation rotation in this material is not accompanied by large elastic distortions. Instead of utilising the Maxwell relation (3), a number of approaches involving combinations of effective-Hamiltonian techniques with molecular dynamics or Monte Carlo simulations have been used to directly compute field-induced temperature and entropy changes in ferroelectrics. ${ }^{47-49}$ (Such techniques can indeed handle polar and elastic spatial inhomogeneities when appropriately parameterised.) Although with no first-order phase transitions present both types of approaches should produce the same results, ${ }^{49}$ the accuracy of the type adopted here may depend on precision of numerical integration of the Maxwell relations. ${ }^{47}$ We have investigated convergence of the integral in Equation (4) for the poling schemes described above, with variations $\leqslant 15-20 \%$ found for the resulting $\Delta T$, as long as step of numerical sweeping of the poling field $\Delta E_{\gamma}$ was not too small (typical step values ranged from 0.5 to $0.05 \mathrm{kV} / \mathrm{cm}$ ).

\section{ACKNOWLEDGEMENTS}

S.N., K.C.P. and J.M. are grateful to the National Science Foundation (DMR 1309114) for partial funding of this project. S.N. also thanks Olle Heinonen and Joseph Mantese for illuminating discussions.

\section{CONTRIBUTIONS}

S.N. and S.P.A. developed the multiscale theoretical framework and supervised the project. S.N. and K.C.P. performed all the DFT simulations. J.M. fitted the Landau-type energy expressions from the DFT results and conducted thermodynamical modeling. J.M., S.N. and S.P.A. co-wrote the manuscript.

\section{COMPETING INTERESTS}

The authors declare no conflict of interest.

\section{REFERENCES}

1. Scott, J. F. Electrocaloric materials. Annu. Rev. Mater. Res. 41, 230-240 (2011).

2. Valant, M. Electrocaloric materials for future solid-state refrigeration technologies. Prog. Mater. Sci. 57, 980-1009 (2012).

3. Alpay, S. P., Mantese, J., Trolier-McKinstry, S., Zhang, Q. \& Whatmore, R. W. Next-generation electrocaloric and pyroelectric materials for solid-state electrothermal energy interconversion. MRS Bull. 39, 1099-1111 (2014).

4. Mischenko, A. S., Zhang, Q., Scott, J. F., Whatmore, R. W. \& Mathur, N. D. Giant electrocaloric effect in thin-film $\mathrm{PbZr}_{0.95} \mathrm{Ti}_{0.05} \mathrm{O}_{3}$. Science 311, 1270-1271 (2006).

5. Chen, H., Ren, T.-L., Wu, X. M., Yang, Y. \& Liu, L.-T. Giant electrocaloric effect in lead-free thin film of strontium bismuth tantalite. Appl. Phys. Lett. 94, 182902 (2009).

6. Bai, Y., Guangping, Z. \& Sanqiang, S. Direct measurement of giant electrocaloric effect in $\mathrm{BaTiO}_{3}$ multilayer thick film structure beyond theoretical prediction. Appl. Phys. Lett. 90, 192902 (2010).

7. Neese, B. et al. Large electrocaloric effect in ferroelectric polymers near room temperature. Science 321, 821-823 (2008).

8. Lu, S.-G. \& Zhang, Q. Electrocaloric materials for solid-state refrigeration. Adv. Mater. 21, 1983-1987 (2009).

9. Lu, S. G. et al. Comparison of directly and indirectly measured electrocaloric effect in relaxor ferroelectric polymers. Appl. Phys. Lett. 97, 202901 (2010).

10. Li, B. et al. Intrinsic electrocaloric effects in ferroelectric poly(vinylidene fluoride-trifluoroethylene) copolymers: Roles of order of phase transition and stresses. Appl. Phys. Lett. 96, 102903 (2010).

11. Qian, X.-S. et al. Large electrocaloric effect in a dielectric liquid possessing a large dielectric anisotropy near the isotropic-nematic transition. Adv. Funct. Mater. 23, 2894-2899 (2013).

12. Correia, T. \& Zhang, Q. Electrocaloric Materials: New Generation of Coolers (Springer-Verlag, 2014).

13. Peng, B., Fan, H. \& Zhang, Q. A giant electrocaloric effect in nanoscale antiferroelectric and ferroelectric phase coexisting in a relaxor $\mathrm{Pb}_{0.8} \mathrm{Ba}_{0.2} \mathrm{ZrO}_{3}$ thin film at room temperature. Adv. Mater. 23, 2987-2992 (2013).

14. Khassaf, H., Mantese, J. V., Bassiri-Gharb, N., Kutnjak, Z. \& Alpay, S. P. Perovskite ferroelectrics and relaxor-ferroelectric solid solutions with large intrinsic electrocaloric response over broad temperature ranges. J. Mater. Chem. C 4, 4763-4769 (2016). 
15. Li, B. et al. The coexistence of the negative and positive electrocaloric effect in ferroelectric thin films for solid-state refrigeration. Europhys. Lett. 102, 47004 (2013).

16. Axelsson, A.-K. et al. Microscopic interpretation of sign reversal in the electrocaloric effect in a ferroelectric $\mathrm{PbMg}_{1 / 3} \mathrm{Nb}_{2 / 3} \mathrm{O}_{3}-30 \mathrm{PbTiO}_{3}$ single crystal. Appl. Phys. Lett. 102, 102902 (2013).

17. Pirc, R., Rožič, B., Koruza, J., Malic, B. \& Kutnjak, Z. Negative electrocaloric effect in antiferroelectric $\mathrm{PbZrO}_{3}$. Europhys. Lett. 107, 17002 (2014).

18. Geng, W. et al. Giant negative electrocaloric effect in antiferroelectric La-doped $\mathrm{Pb}(\mathrm{ZrTi}) \mathrm{O}_{3}$ thin films near room temperature. Adv. Mater. 27, 3165-3169 (2015).

19. Pirc, R., Zutnjak, Z., Blinc, R. \& Zhang, Q. M. Upper bounds on the electrocaloric effect in polar solids. Appl. Phys. Lett. 98, 021909 (2011).

20. Fähler, S. et al. Caloric effects in ferroic materials: new concepts for cooling. Adv. Engr. Mater. 14, 10-19 (2012).

21. Lisenkov, S., Mani, B. K., Change, C.-M., Almand, J. \& Ponomareva, I. Multicaloric effect in ferroelectric $\mathrm{PbTiO}_{3}$ from first principles. Phys. Rev. B 87, 224101 (2013).

22. Liu, Y. et al. Giant room-temperature elastocaloric effect in ferroelectric ultrathin films. Adv. Mater. 26, 6132-6137 (2014).

23. Moya, X., Kar-Narayan, S. \& Mathur, N. Caloric materials near ferroic phase transitions. Nat. Mater. 13, 439-450 (2014).

24. Takeuchi, I. \& Sandeman, K. Solid-state cooling with caloric materials. Phys. Today 68, 48-54 (2015).

25. Goldstone, J., Salam, A. \& Weinberg, S. Broken Symmetries. Phys. Rev. 127, 965-970 (1962).

26. Bruce, A. D. \& Cowley, R. A. Structural Phase Transitions (Taylor and Francis Ltd., 1981)

27. Muševič, I., Blinc, R. \& Žekš, B. The Physics of Ferroelectric and Antiferroelectric Liquid Crystals (World Scientific, 2000).

28. Ruddlesden, S. N. \& Popper, P. New compounds of the $\mathrm{K}_{2} \mathrm{NiF}_{4}$ type. Acta Cryst. 10, 538-539 (1957).

29. Haeni, J. H. et al. Epitaxial growth of the first five members of the $\mathrm{Sr}_{n+1} \mathrm{Ti}_{n} \mathrm{O}_{3 n+1}$ Ruddlesden-Popper homologous series. Appl. Phys. Lett. 78, 3292 (2001).

30. Lee, J. H. et al. Dynamic layer rearrangement during growth of layered oxide films by molecular beam epitaxy. Nat. Mater. 13, 879-883 (2014).

31. Nie, Y. F. et al. Atomically precise interfaces from non-stoichiometric deposition. Nat. Commun. 5, 4530 (2014).

32. Nakhmanson, S. M. \& Naumov, I. Goldstone-like states in a layered perovskite with frustrated polarization: A first-principles investigation of $\mathrm{PbSr}_{2} \mathrm{Ti}_{2} \mathrm{O}_{7}$. Phys. Rev. Lett. 104, 097601 (2010).

33. Bousquet, E., Junquera, J. \& Ghosez, P. First-principles study of competing ferroelectric and antiferroelectric instabilities in $\mathrm{BaTiO}_{3} / \mathrm{BaO}$ superlattices. Phys. Rev. B 82, 045426 (2010).

34. Akcay, G., Alpay, S. P., Rossetti, G. A. \& Scott, J. F. Influence of mechanical boundary conditions on the electrocaloric properties of ferroelectric thin films. J. Appl. Phys. 103, 024104 (2008).

35. Zhang, J., Misirlioglu, I. B., Alpay, S. P. \& Rossetti, G. A. Electrocaloric properties of epitaxial strontium titanate films. Appl. Phys. Lett. 100, 222909 (2012).
36. Strukov, B. A. \& Levanyuk, A. P. Ferroelectric Phenomena in Crystals, Physical Foundations (Springer, 1998).

37. Moya, X. et al. Giant electrocaloric strength in single-crystal $\mathrm{BaTiO}_{3}$. Adv. Mater. 25, 1360-1365 (2013).

38. Lee, B., Nakhmanson, S. M. \& Heinonen, O. Strain induced vortex-to-uniform polarization transitions in soft-ferroelectric nanoparticles. Appl. Phys. Lett. 104, 262906 (2014).

39. Louis, L. \& Nakhmanson, S. M. Structural, vibrational, and dielectric properties of Ruddlesden-Popper $\mathrm{Ba}_{2} \mathrm{ZrO}_{4}$ from first principles. Phys. Rev. B 91, 134103 (2015).

40. Giannozzi, P. et al. QUANTUM ESPRESSO: a modular and open-source software project for quantum simulations of materials. J. Phys. Cond. Matt. 21, 395502 (2009).

41. Perdew, J. P. \& Zunger, A. Self-interaction correction to density-functional approximations for many-electron systems. Phys. Rev. B 23, 5048-5079 (1981).

42. Vanderbilt, D. Soft self-consistent pseudopotentials in a generalized eigenvalue formalism. Phys. Rev. B 41, 7892-7895 (1990).

43. Baroni, S., de Gironcoli, S., Dal Corso, A. \& Giannozzi, P. Phonons and related crystal properties from density-functional perturbation theory. Rev. Mod. Phys. 73, 515-562 (2001).

44. Maradudin, A. A. Theory of Lattice Dynamics in the Harmonic Approximation (Academic Press, 1971).

45. Nakhmanson, S. \& Drabold, D. A. Low-temperature anomalous specific heat without tunneling modes: A simulation for $a$-Si with voids. Phys. Rev. B 61, 5376-5380 (2000).

46. Nakhmanson, S. M., Rabe, K. M. \& Vanderbilt, D. Polarization enhancement in two- and three-component ferroelectric superlattices. Appl. Phys. Lett. 87, 102906 (2005).

47. Ponomareva, I. \& Lisenkov, S. Bridging the macroscopic and atomistic descriptions of the electrocaloric effect. Phys. Rev. Lett. 108, 167604 (2012).

48. Ma, Y.-B., Albe, K. \& Xu, B.-X. Lattice-based monte carlo simulations of the electrocaloric effect in ferroelectrics and relaxor ferroelectrics. Phys. Rev. B 91, 184108 (2015).

49. Marathe, M., Grünebohm, A., Nishimatsu, T., Entel, P. \& Ederer, C. First-principlesbased calculation of the electrocaloric effect in $\mathrm{BaTiO}_{3}$ : $\mathrm{A}$ comparison of direct and indirect methods. Phys. Rev. B 93, 054110 (2016).

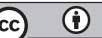

This work is licensed under a Creative Commons Attribution 4.0 International License. The images or other third party material in this article are included in the article's Creative Commons license, unless indicated otherwise in the credit line; if the material is not included under the Creative Commons license, users will need to obtain permission from the license holder to reproduce the material. To view a copy of this license, visit http://creativecommons.org/licenses/ by/4.0/

(c) The Author(s) 2016

Supplementary Information accompanies the paper on the npj Computational Materials website (http://www.nature.com/npjcompumats) 